\title{
$\bullet$ \\ Socio-Cultural Determinants and Diabetes Mellitus in Rural India: A Qualitative Study
}

\section{IJCRR}

Section: Healthcare ISI Impact Factor (2019-20): 1.628 IC Value (2019): 90.81 $\operatorname{SJIF}(2020)=7.893$

(c) (i) (8) Copyright@IJCRR

\section{Shilpa Gaidhane ${ }^{1}$, Quazi Syed Zahiruddin², Mahlaqua Nazli Khatib ${ }^{3}$, Sonali Choudhari², Manoj Patil ${ }^{4}$, Abhay Gaidhane ${ }^{5}$}

\begin{abstract}
'Professor (General Medicine), Faculty in Clinical Epidemiology, School of Epidemiology and Public Health, Jawaharlal Nehru Medical College, Datta Meghe Institute of Medical Sciences, Sawangi (Meghe) Wardha, Maharashtra, India; ${ }^{2}$ rofessor (Community Medicine), Faculty in Global Health, School of Epidemiology and Public Health Jawaharlal Nehru Medical College, Datta Meghe Institute of Medical Sciences, Sawangi (Meghe), Wardha, Maharashtra, India; ${ }^{3}$ Professor (Physiology), Faculty in Evidence Synthesis, School of Epidemiology and Public Health Jawaharlal Nehru Medical College, Datta Meghe Institute of Medical Sciences, Sawangi (Meghe), Wardha, Maharashtra, India; 4 Professor (Community Medicine), Faculty in Global Health, School of Epidemiology and Public Health Jawaharlal Nehru Medical College, Datta Meghe Institute of Medical Sciences, Sawangi (Meghe), Wardha, Maharashtra, India; ${ }^{2}$ Research Consultant, School of Epidemiology and Public Health Jawaharlal Nehru Medical College, Datta Meghe Institute of Medical Sciences, Sawangi (Meghe), Wardha, Maharashtra, India; 5 Director, School of Epidemiology and Public Health Professor (Community Medicine) Jawaharlal Nehru Medical College, Datta Meghe Institute of Medical Sciences, Sawangi (Meghe), Wardha, Maharashtra, India.
\end{abstract}

\section{ABSTRACT}

Introduction: Cultural beliefs, traditional practices and social factors are some of the major determinants of health and disease. So is true for chronic diseases like Diabetes Mellitus requiring long term management.

Objective: To understanding the broader socio-cultural context as important background information for effective care of Diabetics.

Methods: We used 'Explanatory Model Interview Catalogue' interviews of 25 diabetic persons were conducted.

Results: It was found that perception about Diabetes is influenced by traditions, customs and ethos. In Diabetes sign/s don't manifest early and therefore it is usually ignored till it interferes with day to day living of individuals. Following the dietary advice was the most difficult part of diabetes care due to cultural barriers. Additionally, diabetes is so common in society is not considered to be of sufficient priority by the family. Barriers to care-seeking are health illiteracy and cost of care. Diabetics are used to taking complementary treatment along with conventional treatment, which sometimes proves to be hazardous. Language plays a key role in effective diabetes care. Due to socio-cultural factors, females often face problems in receiving appropriate care. Cultural assessment is recommended at initial assessment for identification of cultural issues in care, planning for culturally relevant and acceptable intervention and evaluation.

Conclusion: Cultural values, beliefs, customs, the family pattern may be used as clues for planning diabetes care. The cultural assessment needs to focus on elements relevant to the presenting problem, necessary intervention, and participatory evaluation.

Key Words: Diabetes, Cultural determinants, Barriers for care, Cultural assessment, Rural India

\section{INTRODUCTION}

Globally by 2025 , the adult population will increase by $64 \%$ with more than $35 \%$ of them will develop diabetes, with a consequential increase in people with diabetes by nearly $122 \% .^{1}$ Nearly 51 million people in India are living with Diabetes and this is expected to increase to 87 million by 2030 , accounting for $20 \%$ of the world's population of Diabetics. ${ }^{2,3}$
The last decade saw a rapid increase in the prevalence of diabetes in India. ${ }^{4}$

Optimal glycemic control for a person with diabetes requires him to undertake multiple steps which are complex and integrated, such as dietary modification, medications adherence, regular physical activity, quit tobacco use in any form and regular follow-up for monitor blood glucose. ${ }^{5}$ Social

\section{Corresponding Author:}

Abhay Gaidhane, Director, School of Epidemiology and Public Health, Professor (Community Medicine), Datta Meghe Institute of Medical Sciences, Sawangi (Meghe) Wardha, Maharashtra, India; Phone: +919765404075; Email: abhaygaidhane@gmail.com

ISSN: 2231-2196 (Print)

Received: 24.09 .2020
ISSN: 0975-5241 (Online)

\author{
Revised: 18.11 .2020
}

Accepted: 29.12 .2020
Published: 19.05 .2021 
and cultural belief and traditional practices affect diabetes prevention and care at all levels like perception about diabetes, its assessment and diagnosis, care-seeking behaviour, expectation from the health care system. Since most of these activities are also influenced by a variety of social and cultural factors, therefore, understanding the broader social and cultural context can serve as important background information for effective diabetes care. ${ }^{6}$

Diabetes care providers need to acquire the necessary competencies for assessment and understanding the socio-cultural factors for diabetes prevention and management. ${ }^{7}$ With this background, the present study was conducted to explore the social and cultural issues and their potential influence on diabetes prevention and management in India and to develop a broad framework to guide health care providers for cultural assessment of persons with diabetes.

\section{MATERIALS AND METHODS}

This was a qualitative study, conducted in the rural part of central India. Data were collected by in-depth interviews. Study respondents were 25 people with type II Diabetes Mellitus and currently taking treatment. Written informed consent was taken and confidentiality of information was assured.

We used the Explanatory Model Interview Catalogue (EMIC) semi-structured interviews guides. Respondents were first asked about concepts of health and disease in general and then were asked more specifically about concepts of aetiology and prevention of Diabetes mellitus. Subsequent questions were around social, cultural practices and to catalogue potential influences of these social and cultural practices on diabetes prevention and management in rural India. The questions were open-ended so that the interviewer could probe more on concepts of interest to the study. This approach was used as it is useful in studying the illness meaning as well as understanding the social and cultural factors affecting the care of illness. ${ }^{8}$

Approval was obtained from the Institutional Ethics Committee of DMIMS. Trained Researchers conducted the interviews in the local language (Marathi) for about 30 to 40 minutes. All interviews were recorded, and the recordings were transcribed and coded. Category headings were generated from the data. Two independent researchers verified the apparent accuracy of the category system and after discussion; minor modifications were made to it.

\section{RESULTS}

Among 25 study respondents, 15 were male and 10 were female. Age ranges from 36 years to 62 years and all of them were from rural areas and five were illiterate. All respondents were on anti-diabetic medication.

Respondents mentioned the way people perceive any illness is influenced by their cultural tradition and customs. Respondents highlighted that people in rural areas often try home remedies for ailments, before going to the doctor. Most of the respondents also shared that people believe that diabetes is caused by excessive consumption of sweets and sugar. Particularly chronic illness like diabetes, which shows clinical signs and symptoms or complications in the very late stage of the disease, access to care and treatment is usually ignored till it interferes with their day to day living. One respondent stated that

"We take home remedies when we are sick, but diabetes is not considered a serious illness because it neither shows signs and nor it interferes with day to day work. We tend to ignore the treatment of diabetes even if it is diagnosed, till serious problems occur."

Respondents mentioned they received advice from their doctors for dietary modification. However, many felt that this was the most challenging. Religious festivals and rituals, other social and cultural factors were perceived as the main barriers to following dietary advice. One respondent opined -

"Sweets are prepared during festivals and it is a ritual to serve sweets as Prasad (offerings shared with other devotees after prayer) which we cannot refuse."

Respondents mentioned, in Indian rural areas, usually a common meal is prepared for the entire family and many felt that it is cumbersome to prepare a separate diabetic meal for one single person in the household. Respondents also find it challenging to take frequent meals at short intervals, due to their work pattern in farms. The usual dietary or meal pattern is taking morning and evening tea and two meals in a day and occasionally snacks in the evening. One male respondent expressed -

"I go to the farm for work early in the morning, so mostly, we take morning tea and go to work. I carry my food with me for lunch, which I eat between 1 and 2'o clock in the afternoon. Then I have dinner in the night when I am back at home."

Respondents said the awareness among people from rural areas regarding diabetes was inadequate. Many are not aware of the availability of diabetes diagnosis, treatment and care services. Diabetes does not receive enough priority by the family due to a lack of obvious clinical signs at early stages. Lack of awareness regarding diabetes care and the importance of regular treatment, Indirect cost of care was found to be an important barrier. As per one of the respondents-

"We think why should we see a doctor if we don't feel to be sick (not having sign and symptoms of illness). If we go to the hospital, the entire daytime gets invested in the hospital 
and we lose our wages. We need money to go to hospital and private doctors (doctors from the private sector) charge fees also".

Respondents stated often people with diabetes on conventional anti-diabetes treatment under modern medicine, also use other treatment, like home remedies or some herbal formulations. Participants informed this is a very common practice, but many of those who are on alternative forms of treatment ignores the regular treatment under modern medicine. One participant narrated -

"After getting diagnosed as diabetic 4 years back, I am on regular medication. As per my uncle, excessive consumption of sweets is the cause of diabetes and consuming bitter items help a lot to control blood sugar. I am taking my medications regularly and also drinking Neem juice daily which is too bitter"

Respondents observed often people with diabetes, seeking care felt stressed and not able to follow the advice given by their healthcare providers. One of the reasons mentioned by participants was communication or language barriers and failure of doctors or care providers to understand the social and cultural determinants. One respondent opined -

"Doctors are always in a hurry as they have more patients waiting for them. Many times, we do not understand what he (doctor) said. Doctors in-between speak English or other languages which is difficult for us to understand. The treatment paper (prescription) is also written in English.

Female respondents talked about various social and cultural factors that act as a barrier to access health care. Females ignore their health and lack sufficient support from family for the care of their illness. Males also agree with the challenge's female with diabetes to access care and treatment and follow the advice. One female respondent narrated-

"I work at home only and do not go for farm work. My job is to cook food. I have to take care of my children and husband and consider their choices before cooking foods. I remain engaged for an entire day with the house(hold) works get very little time for myself. My husband is unaware of what drugs I am taking for diabetes, as he is quite busy in his work. Often, I go to the hospital alone.

One more female respondent said

"Amongst all family members in a home, the women usually eat last. For them, it is a bit challenging to follow doctors" advice. The problem is more if there are too many members in the home (joint family).

It was also observed that respondents, even female respondents were not aware of gestational diabetes or diabetes during pregnancy.
Framework to guide health care providers to undertake the cultural assessment to plan culturally appropriate care for people with diabetes

Based on the findings of the present study and literature review the study proposes a framework for cultural assessment. The framework provides a systematic approach for the analysis of beliefs, values, and practices for determining treatment and care needs. ${ }^{9}$ Cultural assessment may be done at various stages of treatment and care (Table 1).

Table 1: Framework for cultural assessment among people with diabetes

Phases Goals $\quad \begin{aligned} & \text { Strategies/topics of } \\ & \text { assessment }\end{aligned}$

General as- To understand the Ethnicity, cross-culture sessment overall social and interaction, religious cultural factors. believes, decision making approaches, communication challenges and barriers to access care

Problem iden- To assess cultural Reasons for accessing tification information spe- care, the stigma associatcifically related to ed with disease (if any), clients presenting perception regarding complaints. the cause of problems, practices related to the treatment / complementary treatment and knowledge about future treatment

Planning and To analyze cul- Clients current and delivering care tural factors that preferred cultural beliefs may influence related to specific care intervention deliv- needs or intervention ery or care plan. planned to deliver.

Evaluation of To plan for care or services delivery and delivered evaluation of the intervention to be delivered.

Interventions need to be co-created. For this patients and family views regarding care or intervention to be incorporated planned for a client, identifying and defining preferred outcome measures to be incorporated into the evaluation.

\section{DISCUSSION}

The study finds out that culture is interpreted and negotiated by people in diverse ways, which influence their beliefs regarding diabetes in a rural community. The various themes that emerged around the influence of cultural factors on diabetes from the study are discussed below. 


\section{Beliefs regarding aetiology treatment and care for people with diabetes}

Sociocultural factors influence how people perceive their overall health, illness and practices related to it. ${ }^{9,10}$ Home treatment, self-medication or the use of herbs for treating illness is deeply rooted in Indian culture. Ritual healings from spiritual healers are not uncommon. Ayurveda, the Indian system of medicine, which is culturally accepted in the Indian community for ages, states that the persons are healthy till their body fluids are in a state of equilibrium or else the illness crop up. Diabetes has been referred to in Ayurveda as a 'Madhumeha' occurring due to inactivity, laziness, lack of exercise, excessive sleep and excessive use of yoghurt, meat and soup of domestic, aquatic and marshy land animals, consumption of unmatured/non-aged grains, products of jaggery and sweets. ${ }^{10,11}$

The study also highlights that sociocultural factors shape the perceptions of people regarding diabetes. Similar to findings in the present study, other studies also reflected on the common perception of Indian people that diabetes is caused due to excessive consumption of sweets. ${ }^{11,12}$ Another misbelief is that consuming bitter vegetables or herbs can reduce blood sugar. ${ }^{13,14}$ Significant risk factors for diabetes mellitus like stress and worry, genetic or hereditary predisposition are ignored. People also believe that smoking and alcohol are not related to diabetes mellitus or its complications. Diabetes is not considered a socially unacceptable or stigmatized disease nor does the disease and its complications are considered significant as it appears very late.

\section{Beliefs around nutrition and dietary habits}

Culture, religion and economic condition play a major role in dietary habits and practices. Oil and sugar are an integral part of the typical Indian diet. Cultural aspects of dietary practices include the identification and methods of food preparation, selection of condiments, time and frequency of meals. Meals symbolize the ritual social aspects. For example, a non-vegetarian diet is strictly not consumed by many communities. Keeping fast is the usual practice among Hindus and Muslims. India is known for a variety of religious festivals. Sweets and high-fat foods are integral representations of festivals and celebrations. Sweets are shared and gifted as a ritual in most festivals.

Dietary management is one of the most important components of a package of care for people with diabetes. This study identified that one of the significant barriers to diabetes care was an inability to practice dietary recommendation due to sociocultural beliefs. In line with dietary habits, a sedentary lifestyle is also a major risk factor for Diabetes. A study by Gaidhane et al., revealed that nearly half of the adolescents had a sedentary lifestyle especially in the age group of 15-19 years. More girls had a sedentary lifestyle compared to boys which were attributed to social and cultural factors. In another study out of 26 diabetics, around 10.4\% had a sedentary lifestyle.

\section{Beliefs regarding care-seeking behaviour}

Disease management decision is closely linked to sociocultural background and resources available. ${ }^{15}$ Sociocultural factors influence access to the health care delivery system and how the family interact with the health care practitioners. ${ }^{9}$ Similar to findings in the present study, other studies also mentioned that even though people and the community do not consider diabetes as a stigmatized disease. Most people do not respond well to diabetes prevention, screening and care programs. The barriers include cultural beliefs, changing priorities and limited access to services. ${ }^{9,10}$ Low health literacy, lack of knowledge related to diabetes services, misconceptions about diabetes, lack of family and social support, lack of patients involvement are some of the determinants influenced by culture responsible for the limited success of the programme. ${ }^{11,12}$ Some misbeliefs result in wasting time by patients on folk medicines. This causes a delay in diabetes care until sign and symptoms of some complications emerge. ${ }^{12-14}$

This study identified that the use of home remedies and herbal formulations is popular among people with diabetes. Similar findings were observed in many other studies. People with diabetes taking conventional modern medicine (allopathic medication) frequently use folk and locally available herbal medicine as home remedies and consider it as either supplements or complementary treatment. ${ }^{15-17}$ Prayer, acupuncture, massage, hot water therapy, biofeedback, and yoga have been frequently used by persons with diabetes ${ }^{16}$. Diabetics consuming juices of bitter herbs is common. ${ }^{13,14}$ The desire for early and maximum benefit are the most common reasons for using these remedies. ${ }^{18} \mathrm{~A}$ clear understanding of these self-care practices is crucial for Health care providers to modify treatment strategies and evaluation of outcomes. Mostly, providers are unaware of patients taking such medication..$^{13}$ It is important for treating physician to be aware that many patients with diabetes may be using complementary medication that may have potential interactions with conventional medicines or have some toxic effect. ${ }^{16}$

The severity of an illness is often judged by people from the amount of pain, disability, and discomfort experienced in routine activities. ${ }^{910}$ People hesitate to seek health care until daily living and functioning are affected. ${ }^{19}$ This study reflects similar findings. Diabetes care providers usually get annoyed with diabetes patients for ignoring blood sugar monitoring which is helpful for early diagnosis and management of complications. Family and social support are highly recommended for adherence to diabetic care. ${ }^{9}$ These factors highly influence effective diabetes care. ${ }^{9,19,20}$ 


\section{Communication between people and diabetes care providers}

Languages and local dialects keep changing across different cultures and societies. These differences also mark a significant barrier in communication. Health literacy is highly influenced by cultural beliefs and education, thereby affecting a person's ability to obtain, interpret, and understand information about health and healthcare service. ${ }^{21}$ Care providers' inability in communicating effectively often results in stress among care providers, poorer client understanding of the disease, less recall of information, decrease client satisfaction and premature termination of care. Our study also reported that language and communication between provider and person with diabetes are critical barriers for diabetes care. Services of a trained translator or bilingual family member can help out in such situations. But the issue of confidentiality needs to be kept in mind. Culturally adapted printed materials in patient's primary language should be given so that the information is available to patients, their family members and other people in their support system. ${ }^{20,21}$ Diabetes care providers, to ensure effective communication with persons with diabetes needs to present themselves as a colleague, establish ties with family and friend of patients, supportive and personalized approach, demonstrating respect, avoiding confrontation and use therapeutic silence and touch. ${ }^{22}$ In a study by Gaidhane et al, 2014 assessing the challenges of primary health care providers, it was found that lack of clear guidelines $(92.2 \%)$, lack of facility for investigations (69\%), limited supply of drugs ( $68 \%$ ), the perception that diabetes is difficult to manage at primary care level $(93.1 \%)$ and low confidence of patients in primary care level $(72.4 \%)$ were some challenges for diabetes care at primary care level. ${ }^{24,25}$

\section{Women and diabetes}

Women are often considered as the custodian of family values and culture in India. This responsibility to maintain deeply rooted social and cultural practices and pass them on to younger generations can make it difficult for them to successfully make lifestyle changes leading to poor health outcomes. ${ }^{23}$ This study revealed that women with diabetes find it challenging to follow the advice of diabetes care provider due to these social and cultural practices. Women find it difficult to follow the care advice given by doctors and selfmedication is very common in them. Furthermore, around $7 \%-17 \%$ of women of childbearing age are reported to have gestational diabetes ${ }^{24,25}$. Effective diabetes management during pregnancy is highly influenced by cultural beliefs, practices and the overall status of women in the family. ${ }^{23}$

\section{CONCLUSION}

To conclude, improving diabetes care needs a better understanding of the sociocultural determinants. To address these issues in prevention and care services for diabetes providers needs competencies for assessment and planning socially and culturally appropriate interventions. To deliver these culturally appropriate intervention and effective continuum of diabetes care needs innovative models with the multidisciplinary team, including the lay caregiver. These specific interventions are well aligned with the local context and likely to have a significant impact on diabetes care. However, such models need to be tested and evaluated. Respondents knowledge and beliefs about Diabetes and its care found to be determined by their tradition, customs and ethos observed in their society for generations. Hence, cultural values, beliefs, customs, the family pattern may be used as clues for planning diabetes care. Such interventions are likely to have a significant impact on overall diabetes care. The cultural assessment needs to focus on elements relevant to the presenting problem, necessary intervention, and participatory evaluation.

\section{Conflict of Interest: Nil}

\section{Funding: Nil}

\section{Authors contribution:}

Shilpa Gaidhane: data collection and manuscript preparation

Quazi Syed Zahiruddi: Conceptualization of topic Data analysis, manuscript preparation

Mahlaqua Nazli Khatib: Data collection, manuscript writing and editing

Sonali Choudhari: Data interpretation, manuscript writing and editing

Manoj Patil: Data interpretation, manuscript writing and editing

Abhay Gaidhane: Conceptualization of topic, preparation of tools, Data analysis, manuscript preparation

\section{REFERENCES}

1. King H, Aubert RE, Herman WH. Global burden of diabetes, 1995-2025: prevalence, numerical estimates, and projections. Diabetes Care 1998;21(9):1414-1431.

2. Sicree R, Shaw J, P Z, editors. Diabetes and impaired glucose tolerance. In D. Gan (Ed)., Diabetes Atlas. International Diabetes Federation. Third Edition, Published by International Diabetes Federation, Belgium, 2006; 5(15):103-106.

3. Mohan V, Vassy J, Pradeepa R, Deepa M, Subashini S. The Indian Type 2 Diabetes Risk Score also Helps Identify those at Risk of Macrovascular Disease and Neuropathy (CURES-77). JAPI 2010;58:430-433.

4. Mohan V, Sandeep S, Deepa R, Shah B, Varghese C. Epidemiology of type 2 diabetes: Indian scenario. Indian J Med Res 2007; 125:217-230.

5. Heisler M, Smith DM, Hayward RA, Krein SL, Kerr E. How well do patients' assessments of their diabetes self-management correlate with actual glycemic control and receipt of recommended diabetes services? Diabetes Care 2003;26:738-743. 
6. Tripp-Reimer T, Choi E, Kelley LS, Enslein JC. Cultural Barriers to Care: Inverting the Problem. Diabetes Spectr 2001;14(1):1322.

7. Osman A, Curzio J. South Asian cultural concepts in diabetes. Nurs Times 2012;108(10):30-32.

8. Weiss M. Explanatory Model Interview Catalogue (EMIC): Framework for Comparative Study of Illness. Transcult Psychiatry 1997;34:235-263.

9. Tripp-Reimer T, Choi E, Kelley LS, Enslein JC. Cultural Barriers to Care: Inverting the Problem. Diabetes Spectrum 2001;14(1):13-22.

10. Campos C. Narrowing the Cultural Divide in Diabetes Mellitus Care: A Focus on Improving Cultural Competency to Better Serve Hispanic/Latino Populations. Insulin 2006;1(2):70-76.

11. WHO Expert Committee. Physical status: use and interpretation of anthropometry data. WHO Technical Report Series 854. World Health Organisation, Geneva 1995.

12. Alhyas L, McKay A. Socio-cultural aspects of diabetes care: Myths about diabetes in Qassim region, Saudi Arabia. Anna Alq Med 2010;6:1431.

13. Singh J, Singh R, Gautam CS. Self-medication with herbal remedies amongst patients of type 2 diabetes mellitus: A preliminary study. Ind J Endocrinol Metab 2012;16(4):662-663.

14. Sushama S, Nandita T. Study on Self-Medication and Self DietManagement by Women of Indore City, India. Res J Recent Sci 2012;1:354-356.

15. Chacko E. Culture and therapy: complementary strategies for the treatment of type-2 diabetes in an urban setting in Kerala, India. Soc Sci Med 2003;56(5):1087-1098.

16. Dham S, Shah V, Hirsch S, Banerji MA. The role of complementary and alternative medicine in diabetes. Curr Diab Rep 2006;6(3):251-258.
17. Sethi A, Srivastava S, Madhu SV. Prevalence and pattern of use of indigenous medicines in diabetic patients attending a tertiary care centre. J Indian Med Assoc 2011;109(7):469-471.

18. Mehrotra R, Bajaj S, Kumar D. Use of complementary and alternative medicine by patients with diabetes mellitus. Natl Med J Ind 2004; 17(5):243-245.

19. Lawton J, Ahmad N, Hallowell N, Hanna L, Dougla M. Perceptions and experiences of taking oral hypoglycaemic agents among people of Pakistani and Indian origin: a qualitative study. Br Med J 2005; 3(2):1134-1136

20. Tripp-Reimer T: Cultural assessment. In Nursing Assessment: A Multidimensional Approach. Bellack J, Bamford P, Eds. Monterey, Calif., Wadsworth Health Sciences, 1984: 226-246

21. Greenhalgh T. Barriers to concordance with antidiabetic drugs--cultural differences or human nature? $\mathrm{Br}$ Med J 2005;330(7502):1250.

22. Lawson B, Aarsen KV, Latter C, Putnam W, Natarajan N, Burge F. Self-Reported Health Beliefs, Lifestyle and Health Behaviours in Community-Based Patients with Diabetes and Hypertension. Canad J Diab 2011;35(5):490-496.

23. Black SA. Diabetes, Diversity, and Disparity: What Do We Do With the Evidence? Ame J Public Health 2002;92:543-8.

24. Wahi P, Dogra V, Jandial K, Bhagat R, Gupta R, Gupta S, et al. Prevalence of gestational diabetes mellitus (GDM) and its outcomes in Jammu region. J Assoc Physicians Ind 2011;59:227230.

25. Balaji VS, Balaji MS, Sanjeevi C, Green A. Gestational Diabetes Mellitus in India. J Assoc Phys Ind 2004;52:707. 